\title{
Information and the memory span
}

\author{
LESTER M. HYMAN, STANFORD UNIVERSITY \\ HERBERT KAUFMAN, UNIVERSITY OF CONNECTICUT
}

Messages differing in number of symbols and symbol information load were presented tachistoscopically to 4 adult Ss. The messages were constructed by random drawing with replacement from an alphabet of 8 black form symbols and an alphabet of 32 colored form symbols. The number of symbols recalled varied as a function of alphabet; however, the information in recall was constant for all conditions. The number of symbols recalled and the information in recall was independent of message length.

The immediate memory span or the "span of attention" has been of interest to psychologists for many years. Traditionally, the immediate memory span has been reported in terms of the number of symbols (words, letters, numbers, figures, etc.) that Ss could correctly recall (Glanville \& Dallenbach, 1929). Such researchers have found that for many types of symbols the number of items recalled is a constant, usually approximately equal to 7 . In the past decade the number of bits of information in the symbols recalled has been reported along with the more traditional measurements of immediate memory span. A comparison of these measurements led Miller to the conclusion that, "the number of chunks of information is constant for immediate memory"' (1956, p. 92).

Miller drew supporting evidence for his statement from experiments using sequential presentation of familiar visual symbols (Pollack, 1953; Hayes, 1952). The evidence from experiments using simultaneous presentation of familiar and unfamiliar visual symbols lends less compelling support to Miller's statement. Variables found to affect the span of immediate memory in the simultaneous presentation experiments include: the information load of the symbols (Glanville \& Dallenbach, 1929), the number of symbols presented (Anderson \& Fitts, 1959; Krulee, 1959), the identifiability of the symbols (Anderson \& Fitts, 1959), and time considerations in the stimulus presentation (Cherry, 1961). Sperling's use of a post-stimulus cueing technique has emphasized the importance of the period of time following stimulus presentation in extracting information from immediate memory (Sperling, 1960). Although stimulus-response compatibility has not been shown to affect memory span, Fitts and Switzer (1962) have shown it to be an important variable affecting information transmission in reaction time studies.

This study differs from previous studies in two ways. First, it allows for a comparison of the effects on immediate memory of relatively unfamiliar symbols differing in information load; second, it allows comparison of the effects of different message lengths (number of symbols per presentation) on immediate memory using unfamiliar symbols.

\author{
METHOD \\ Stimuli, Apparatus and Subjects. \\ Two sets of symbols were formed to give two "alpha-
} bets" differing in information content. The "low" information alphabet contained eight black geometric forms and was called the " $F$ "'alphabet. The forms used were triangle, star, heart, diamond, square, circle, $T$ and $X$. Four colorings each of these eight forms yielded the 32 symbols of the "high" information or "CF"' alphabet. The four colorings were blue, red, green and copper. Messages were formed from each of the alphabets by random sampling with replacement. Messages composed of symbols from the " $F$ "' alphabet contained three bits of information per symbol while messages composed with symbols from the "CF"" alphabet contained five bits of information per symbol. To allow overlap of both number of symbols per message and the total message information content, messages of four, six and eight symbols were constructed from the " $F$ " alphabet and messages of four and six symbols were constructed from the "CF"' alphabet. Fifty messages of each length for each alphabet were constructed. The symbols, $3 / 4$ in. high by $3 / 4$ in. wide were painted on $8-1 / 2 \times 11$ in. white cards. Four-symbol messages appeared as one row of four characters with $3 / 4$-in. spaces between adjacent symbols. Six-symbol messages appeared as two rows of three symbols each and eight-symbol messages appeared as two rows of four symbols each. The messages were centered on the cards with $3 / 4$-in. spacing between rows.

The cards were presented in a two-field Gerbrands tachistoscope. The second field contained a center fixation point and was brightly lit to minimize afterimages. Four graduate students in psychology served as volunteer Ss.

\section{Procedure}

Prior to the first session the Ss examined all the symbols used in the experiment and chose a set of response symbols to use. All Ss agreed to use the symbols $\Delta, S, H, D, \square, O, T, X$ to indicate the forms of presented figures and $R, B, G$, and $C$ to indicate the color of the "CF" symbols. Responses were made on a grid containing boxes that corresponded to the number and positions of the symbols of the messages presented.

The Ss were seated at the tachistoscope in a darkened room and asked to fixate on the fixation point. They were told which set of symbols would appear and what message length would be shown. When the S said, "ready," a stimulus card was flashed on the screen for a brief period of time. The $\mathrm{S}$ was then given as much time as 


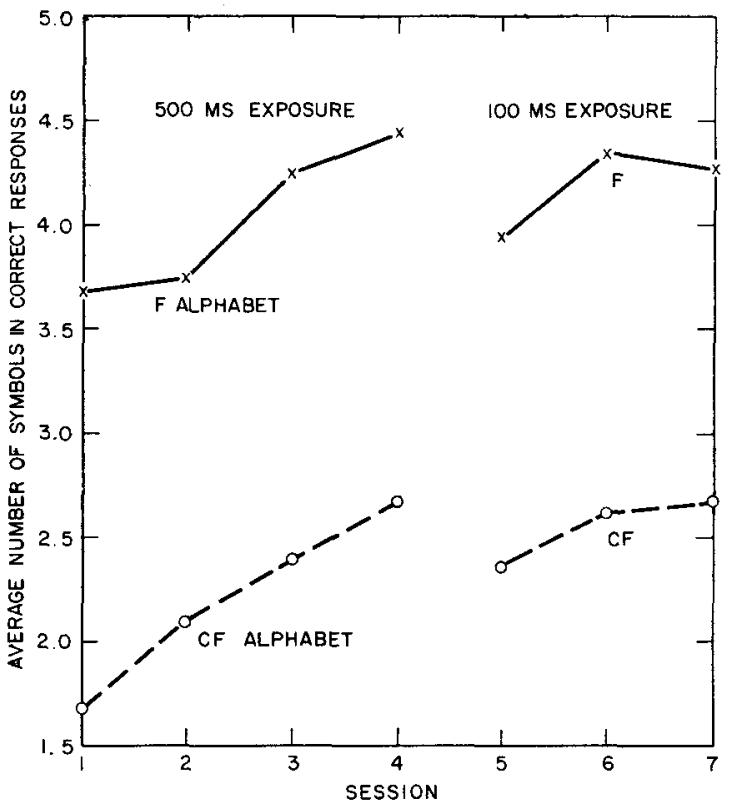

Fig. 1. Number of symbols correctly recalled. Each point represents the average recall of $\mathbf{4 0 0}$ messages.

he wished to write down the symbols on the response grid while a new message was readied for presentation. The procedure was repeated for the 50 messages of the message length and alphabet being presented. The presentation of the 50 messages of a given message length from a given alphabet constituted a session; a $\mathrm{S}$ sat through no more than one experimental session per day.

\section{Experimental Design.}

The experiment consisted of two parts. In the first part the Ss were exposed to the $4 \mathrm{~F}, 8 \mathrm{~F}, 4 \mathrm{CF}$ and $6 \mathrm{CF}$ messages. Each message was exposed for $500 \mathrm{msec}$. The Ss received each set of messages for four sessions, and the order of presentation of the different message types was balanced among the Ss. The order of messages within message types was randomized each time a message type was re-presented to a given $S$. During the second part of the experiment the Ss were exposed to the $6 \mathrm{~F}, 8 \mathrm{~F}, 4 \mathrm{CF}$ and $6 \mathrm{CF}$ messages, each message being presented for $100 \mathrm{msec}$. Each message type occurred three times and the order of appearance of conditions was again balanced among the Ss.

\section{RESULTS}

Number of Symbols Recalled.

Figure 1 presents the number of symbols correctly recalled as a function of session for the two alphabets averaged over message length and Ss. Responses to messages of different length were averaged when an analysis of variance showed no significant differences due to this variable $(F=.317, d f=1 / 12, p>.1)$. Since the number of symbols correctly recalled was averaged over message length, each point on Fig. 1 represents data from two sessions, one with the shorter and one with the longer messages. Each $\mathrm{S}$ sat for 28 sessions. An analysis of variance including data from all the $100 \mathrm{msec}$. exposure sessions showed a highly significant difference between the number of symbols recalled from the two alphabets $(F=90.25, \mathrm{df}=1 / 12, \mathrm{p}<.001)$.

\section{Amount of Information in the Symbols Recalled.}

Figure 2 presents the amount of information in the symbols correctly recalled for the two alphabets. As in Fig. 1 the data a re averaged over message length and Ss. Although there is some initial difference in the information in the symbols correctly recalled between the alphabets, this difference becomes negligible by the last session of the $500 \mathrm{msec}$. exposure, and remains small over the remaining sessions. By the end of the last $500 \mathrm{msec}$. exposure session all Ss had reached near perfect performance with the $4 \mathrm{~F}$ messages. As a result the $4 \mathrm{~F}$ messages were not presented during the shorter exposure time sessions. At the end of the experiment the Ss were given one additional session with the $4 \mathrm{~F}$ messages at the $100 \mathrm{msec}$. exposure time and their performance was still near perfect. An analysis of variance on the data from the $100 \mathrm{msec}$. exposure time sessions showed no significant difference between the amount of information in the symbols correctly recalled for the two alphabets $(F=.395, d f=1 / 12, p>.1)$.

Additional analyses were done to determine the effect of symbols recalled in incorrect positions and of partial recall of the CF symbols. Again there were significant differences between the number of symbols recalled for the two alphabets and no difference in the amount of information in that recall.

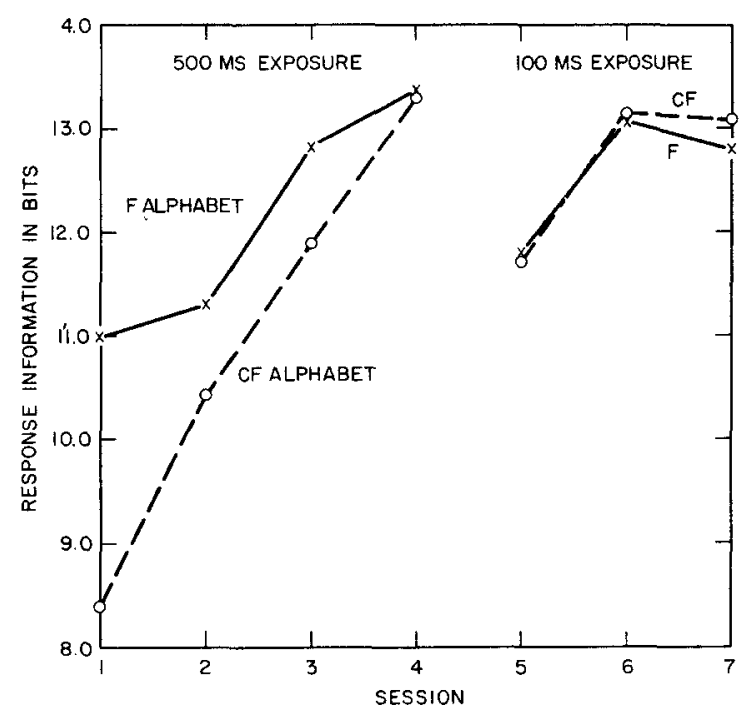

Fig. 2. Information content in bits of the symbols correctly recalled. Each point represents the average of 400 messages. 


\section{DISCUSSION}

It would seem that under the conditions used in this experiment with the relatively unitary symbols presented, immediate memory is relatively constant for information and not constant for number of symbols. Since partially correct responses were possible and did occur, one possible explanation is that the symbols were not unitary and do not fall into the category of Miller's "chunks." This criticism is reasonable but cannot account for the constancy of information transmission found when partially correct responses were analyzed. An alternative explanation of the difference between the results of this experiment and the experiments on which Miller based his "chunking" hypothesis might be found in the human's ability to code stimuli. Simultaneous presentation of symbols minimizes the time available for a $\mathrm{S}$ to encode them. If the symbols are familiar and a set of responses available which have a high stimulus-response compatibility, Ss may still be able to encode them during the brief interval that a stimulus trace remains. There was evidence in the data from this experiment that some "fast" coding does take place. Many of the messages in both alphabets contained more than one instance of a given symbol. An additional analysis showed that symbol recall and information transmission was greater as a fairly direct function of the number of repeated symbols in a message. Sequential presentation of a set of symbols offers the $\mathrm{S}$ a relatively large amount of time to encode visual stimuli into "chunks" containing larger amounts of information. Using the reasonable assumption that encoding information into "chunks" takes time, the results of this experiment are not necessarily inconsistent with Miller's "chunking" hypothesis but rather indicate a (temporal) bound on conditions under which the hypothesis holds. Corroborating evidence for this assumption has just become available from a recent study by Lamb (1966) which showed a perfect trade-off between information storage as a function of code redundancy and processing time. An experiment which compares the results of sequential and simultaneous presentation of unfamiliar symbols is planned as an additional test of the "coding time" explanation of the results found here.

\section{References}

Anderson, N. S., \& Fitts, P. M. Amount of information gained during brief exposure of numerals and colors. Readings for human engineering, concepts and theory. University of Michigan, $1959,12.5-1$ to $12.5-8$.

Attneave, F. Applications of information theory to psychology. New York: Holt, 1959

Cherry, C. On human communication. New York: Science Editions Inc., 1961

Fitts, P. M., \& Switzer, G, Cognitive aspects of information processing. J. exp. Psychol., 1962, 63, 321-329.

Glanville, A. D., \& Dallenbach, K. M. The range of attention. Amer. J. Psychol., 1929, 41, 207-236.

Hayes, J. R. M. Memory span for several vocabularies as a function of vocabulary size. Quarterly Progress Report. Cambridge Mass: Acoustics Laboratory, Massachusetts Institute of Technology, Jan.-June, 1952.

Krulee, G. K. Information theory and man machine systems. Readings for human engineering, concepts and theory. University of Michigan, 1959, 12.1-1 to 12.1-9.

Lamb, J. C. Codes and human channel capacity. Unpublished Ph.D. thesis, University of Connecticut, 1966.

Miller, G. A. The magical number seven, plus or minus two. Some limits of our capacity for processing information. Psychol. Rev. $1956,63,81-97$.

Pollack, I. The assimilation of sequentially encoded information. Amer. J. Psychol., 1953, 66, 421-435.

Sperling, G. The information available in brief visual presentations. Psychol. Monogr., 1960, 74, No. 11, 1-29.

(Received in the Editorial Office May 19, 1966.) 